\title{
Regenerative medicine therapy for single ventricle congenital heart disease
}

\author{
Chetan Ambastha, Gregory J. Bittle, David Morales, Nathaniel Parchment, Progyaparamita Saha, \\ Rachana Mishra, Sudhish Sharma, Alexander Vasilenko, Muthukumar Gunasekaran, Manal T. Al-Suqi, \\ Deqiang Li, Peixin Yang, Sunjay Kaushal
}

Division of Cardiac Surgery, University of Maryland School of Medicine, Baltimore, MD, USA

Contributions: (I) Conception and design: C Ambastha, GJ Bittle, D Morales, D Li, S Kaushal; (II) Administrative support: C Ambastha, GJ Bittle; (III) Provision of study materials or patients: C Ambastha, GJ Bittle, D Morales, N Parchment, P Saha, R Mishra, S Sharma, A Vasilenko, M Gunasekeran, MT Al-Suqi, D Li, S Kaushal; (IV) Collection and assembly of data: C Ambastha, GJ Bittle, D Morales, N Parchment, D Li, S Kaushal; (V) Data analysis and interpretation: C Ambastha, GJ Bittle, D Morales, N Parchment, P Saha, R Mishra, S Sharma, A Vasilenko, M Gunasekeran, D Li, S Kaushal; (VI) Manuscript writing: All authors; (VII) Final approval of manuscript: All authors.

Correspondence to: Sunjay Kaushal, MD, PhD. Associate Professor of Surgery, Director, Pediatric and Adult Congenital Surgery, 110 S. Paca St., $7^{\text {th }}$ Floor, Baltimore, MD 21201, USA. Email: skaushal@som.umaryland.edu.

\begin{abstract}
One of the most complex forms of congenital heart disease (CHD) involving single ventricle physiology is hypoplastic left heart syndrome (HLHS), characterized by underdevelopment of the left ventricle (LV), mitral and aortic valves, and narrowing of the ascending aorta. The underdeveloped LV is incapable of providing long-term systemic flow, and if left untreated, the condition is fatal. Current treatment for this condition consists of three consecutive staged palliative operations: the first is conducted within the first few weeks of birth, the second between 4 to 6 months, and the third and final surgery within the first 4 years. At the conclusion of the third surgery, systemic perfusion is provided by the right ventricle (RV), and deoxygenated blood flows passively to the pulmonary vasculature. Despite these palliative interventions, the $\mathrm{RV}$, which is ill suited to provide long-term systemic perfusion, is prone to eventual failure. In the absence of satisfying curative treatments, stem cell therapy may represent one innovative approach to the management of RV dysfunction in HLHS patients. Several stem cell populations from different tissues (cardiac and noncardiac), different age groups (adult- vs. neonate-derived), and different donors (autologous vs. allogeneic), are under active investigation. Preclinical trials in small and large animal models have elucidated several mechanisms by which these stem cells affect the injured myocardium, and are driving the shift from a paradigm based upon cellular engraftment and differentiation to one based primarily on paracrine effects. Recent studies have comprehensively evaluated the individual components of the stem cells' secretomes, shedding new light on the intracellular and extracellular pathways at the center of their therapeutic effects. This research has laid the groundwork for clinical application, and there are now several trials of stem cell therapies in pediatric populations that will provide important insights into the value of this therapeutic strategy in the management of HLHS and other forms of CHD. This article reviews the many stem cell types applied to CHD, their preclinical investigation and the mechanisms by which they might affect RV dysfunction in HLHS patients, and finally, the completed and ongoing clinical trials of stem cell therapy in patients with CHD.
\end{abstract}

Keywords: Heart defects; congenital; heart failure; hypoplastic left heart syndrome (HLHS); regenerative medicine; stem cells

Submitted Mar 05, 2018. Accepted for publication Apr 03, 2018.

doi: 10.21037/tp.2018.04.01

View this article at: http://dx.doi.org/10.21037/tp.2018.04.01 


\section{Introduction}

Hypoplastic left heart syndrome (HLHS) is the most common form of single ventricle congenital heart disease (CHD), with a prevalence of 2 to 3 per 10,000 live births (1-3), and is defined by underdevelopment of the left ventricle (LV), which is unable to support systemic perfusion. Without treatment, this condition is fatal. The abnormal anatomy in HLHS can, however, be surgically modified to one that is life-sustaining which requires three staged palliative surgeries. The stage I operation (Norwood) is the most complex of the three, and carries the highest rate of post-operative morbidity and mortality (4-6). It is performed within the first days to weeks after birth, and commits the right ventricle (RV) to systemic circulation. Pulmonary blood flow is supplied by a systemic arterial to pulmonary arterial shunt (modified Blalock-Taussig, mBT), or an RV to pulmonary artery (PA) (Sano) shunt (4). Each of these shunts presents its respective challenges, with the mBT shunt potentially resulting in coronary insufficiency, whereas the Sano shunt may cause arrhythmias or RV fibrosis. During the stage II (Glenn) and stage III (Fontan) palliative operations, the systemic to PA shunt is taken down, then the superior vena cava (SVC) and inferior vena cava (IVC), respectively, are directly anastomosed to the PA to provide pulmonary perfusion. Following Fontan completion, deoxygenated venous blood drains passively through the pulmonary vasculature.

Although heroic, these surgeries are not curative, and long-term impediments to survival and quality of life persist even following palliation. These are primarily related to gradual, and ultimately fatal, right ventricular dysfunction secondary to protracted subjection to systemic afterload. Unlike the LV, the structure and gene expression profile of the RV render it ill-equipped for prolonged exposure to systemic pressures $(7,8)$. The RV's thinner walls and crescent shape allow it to adapt to large changes in beat-tobeat volume loading, such as during respiration or changes in position, but are less robust to pressure overload than the thick walls and bullet shape of the LV (7-10). Chronic RV pressure overload can also lead to an early and detrimental switch from fatty acid metabolism to glycolysis (8), cardiomyocyte hypertrophy, reduced myocardial capillary density (11), and myocardial fibrosis $(7,8)$, which are exacerbated by the volume overload experienced during the interstage period after the Norwood operation, but prior to the Fontan.

Because of the particular mechanisms and kinetics of RV failure in single ventricle CHD, therapies designed for $\mathrm{LV}$ dysfunction may not be effective. Clinical trials employing drugs including beta blockers, angiotensin-converting enzyme (ACE) inhibitors, and angiotensin II receptor blockers (ARBs), have demonstrated mixed or inconclusive results when applied to patients facing RV failure $(8,11)$. Currently, the only therapeutic option to intractable heart failure following staged palliation is heart transplantation. Although long-term survival for Fontan patients following heart transplantation is comparable to other CHD populations, early post-transplant mortality is greater in the Fontan group $(12,13)$.

In the absence of a satisfying treatment for failure of the single RV, novel therapeutic approaches are under investigation for this challenging clinical issue. Stem cellbased therapeutics may satisfy such a niche, as they have been associated with favorable functional outcomes in a number of preclinical models of heart failure, and are now being shown to improve cardiac performance and quality of life in early pediatric cardiac clinical trials. In this review, we will define the different stem cell types currently being studied as cardiac therapeutics, describe preclinical and clinical trials using stem cell therapies in CHD patients, and address the potential application and mechanism of action of these therapies as applied to CHD patients.

\section{Stem cell types}

\section{Mesenchymal stem cells (MSCs) (bone marrow-derived)}

MSCs are a population of stem cells that are derived from bone marrow stromal cells, and are capable of differentiating into mesodermal tissues, including bone, cartilage, muscle, tendon, ligament, and adipose tissue (14). MSCs can also be isolated from other sources, such as umbilical cord blood (UCB) (discussed in the next section), adipose tissue, and peripheral blood samples (15). MSCs have unique immunological attributes, as they have reduced expression of MHC class I and lack MHC class II and costimulatory molecules CD80 (B7-1), CD86 (B7-2), and CD40 (14). These unique immunomodulatory properties have allowed in vivo evaluation of both autologous and allogenic MSC preparations, and likely contribute to their promotion of favorable cardiac remodeling after myocardial infarction (MI) in animal models $(16,17)$.

Since their landmark modern description 1990s, MSCs have been the subject of over 20,000 publications and 32 clinical trials for adult diseases including acute MI, ischemic cardiomyopathy, and non-ischemic dilated cardiomyopathy. These trials most-commonly utilized 
bone marrow-derived MSCs, but several trials evaluated MSCs from other sources, such as UCB. The earliest trials focused on MSCs of autologous origin, but this approach was limited by the 4 to 6 weeks requirement to culture a therapeutic dose, prompting the investigation of allogeneic MSC preparations. In the phase I, double-blind, placebocontrolled Safety Study of Adult Mesenchymal Stem Cells to Treat Acute Myocardial Infarction (NCT00114452), 53 patients underwent intravenous administration of allogeneic MSCs or placebo within 10 days after acute MI (18). Though it was designed to assess safety, this study demonstrated improved functional outcomes in the allogeneic MSC-treated patients, including a reduction in ventricular arrhythmias, improved pulmonary function, improved left ventricular ejection fraction (LVEF) in patients with anterior MI, and improved quality of life at 6-month follow-up. The efficacy of allogenic MSCs was subsequently redemonstrated in the phase I/II Percutaneous Stem Cell Injection Delivery Effects on Neomyogenesis Pilot Study (POSEIDON)-Pilot trial (NCT01087996) and POSEIDON-DCM trial (NCT01392625), which focused on adults with either ischemic or non-ischemic cardiomyopathy. MSCs were administrated directly into the LV myocardium by catheter-based transendocardial injection. Among patients with ischemic disease (POSEIDON-Pilot trial), MSC administration was associated with acceptably low rates of adverse events for both the allogenic and autologous groups. While patients who received autologous MSCs demonstrated some improvement in 6 minute walk test distance and Minnesota Living with Heart Failure Questionnaire (MLHFQ) scores, neither group showed a significant change in cardiac function (19). Among patients with non-ischemic disease (POSEIDON-DCM trial), allogeneic MSC administration was associated with a significant increase in LVEF and 6-minute walk test distance in comparison to the autologous group (20). One patient out of the 19 in the allogeneic cohort did develop donor-specific antibodies. Taken together, these early clinical trials with allogeneic MSCs have demonstrated safety and clinically meaningful efficacy.

\section{UCB-derived cells}

UCB is a source of both hematopoietic and nonhematopoietic precursors that were initially used in the treatment of hematologic disorders (21). MSCs can also be isolated from UCB (UCB-MSC), and these cells can be differentiated into classical MSC lineages (bone, cartilage, and fat), as well as hepatocyte-like cells, neuroglial-like cells (22), respiratory epithelial cells (23), and cardiomyocytes (24). In preclinical studies, transplanted UCB-MSCs have been shown to improve left ventricular structure and function following MI (25) and following right ventricular structure and function following induction of acute pressure overload (26), with tissue-level evidence of increased angiogenesis and decreased myocardial fibrosis (27). The only completed clinical trial using UCBs is the phase I/II, double-blind, controlled Randomized Clinical Trail of Intravenous Infusion of Umbilical Cord Mesenchymal Stem Cells on Cardiomyopathy trial (RIMECARD, NCT01739777) (28). This trial evaluated the intravenous administration of allogeneic UMB-MSCs in adults with chronic heart failure with reduced ejection fraction [New York Heart Association (NYHA) classes I-III, LVEF $\leq 40 \%]$. UCB-MSC administration was associated with a significant increase in LVEF at 1 year compared to control $(+7 \% v s .+2 \%)$, and a decrease in heart failure symptoms as assessed by NYHA class and MLHFQ score. Seven of the 15 UCB-MSCtreated patients were evaluated for anti-donor antibodies, but no such antibodies were detected.

\section{Cardiosphere-derived cells (CDCs)}

Cardiospheres are heterogeneous, self-assembling spherical cellular clusters which arise from myocardial tissue cultured on poly-D-lysine (29). These cellular structures are composed of a core of undifferentiated cells surrounded by a shell of cardiac-committed cells (30). CDCs are produced by expanding cardiospheres on fibronectin-coated plastic. The heart tissue from which CDCs can be isolated and expanded is typically obtained by endomyocardial biopsy, but may also be collected during open cardiac surgical interventions. Like MSCs, the administration of allogeneic CDCs has been shown to be safe, and has successfully promoted cardiac regeneration and improved cardiac function in animal models of MI $(31,32)$.

The first clinical evaluation of CDCs was conducted as the Cardiosphere-Derived Autologous Stem Cells to Reverse Ventricular Dysfunction (CADUCEUS, NCT00893360) trial (33). Seventeen adult patients with left ventricular dysfunction after recent MI were treated by intracoronary CDC administration, then compared to 8 similar patients who did not receive cells. There were no treatment-related arrhythmias or other safety concerns, and CDC administration was associated with modest improvement in LVEF with marked reduction in myocardial 
scar formation. More recently, an allogeneic CDC administration was evaluated in the phase I/II, randomized, double-blind, placebo-controlled Allogeneic Heart Stem Cells to Achieve Myocardial Regeneration (ALLSTAR, NCT01458405) trial (34). This 142 -patient trial was designed to detect a decrease in infarct size in adults at 12 months post-MI, but was stopped after a scheduled 6-month interim analysis identified a low probability of identifying a significant difference in this endpoint. Considering only the partial trial data, the treatment group reported a borderline-significant $(\mathrm{P}=0.05)$ reduction in mean LV end-diastolic volume, as well as a trend towards reduction of mean $\mathrm{LV}$ end-systolic volume.

\section{C-kit ${ }^{+}$cardiac progenitor cells (CPCs)}

CPCs are resident cardiac stem that are characterized by their expression of c-kit, a surface receptor tyrosine kinase. Unlike hematopoietic cells, CPCs lack expression of CD45 or Lin (general hematopoietic markers), or tryptase (mast cell-specific marker). Several investigators have shown that CPC administration effectively attenuates $L V$ dysfunction in preclinical models of both acute and chronic myocardial ischemia $(35,36)$.

As resident cardiac cells, the natural density of CPCs in the myocardium and their performance in functional assays has been clearly delineated in CHD patients $(37,38)$. CPCs were isolated from right atrial appendage samples derived from patients of different ages undergoing surgical procedures for CHD. CPC density within the myocardium was shown to decrease with age, falling from $9 \%$ in neonates to approximately $3 \%$ in older children (37). The presence of symptomatic heart failure (NYHA class III or IV) was associated with maintenance of neonatal-level CPC density within the myocardium regardless of age (up to 14 years). CPC densities in other regions of heart are known to be considerably lower than those observed in the right atrium, so these numbers likely represent an overestimate of the prevalence of this cell type (39).

In addition to being present in the greatest numbers, neonatal CPCs (nCPCs) have been shown to possess superior therapeutic potency with respect to adult CPCs (aCPCs) in a rat MI model. nCPC administration was associated with preservation of LVEF at 7 days (nCPC $71 \%$ vs. aCPC $63 \%$ ) and 28 days (nCPC $69 \%$ vs. aCPC 60\%) post-injection, with histologic evidence of decreased periinfarct inflammation and fibrosis (40). These findings have been reproduced in a rat model of pressure-induced RV dysfunction (main PA banding) (41). This study evaluated human CPCs isolated from three different age groups: neonates ( $0-1$ month of age), infants ( 1 month-1 year of age), and children (1-5 years of age). At 2 weeks postbanding/injection, rats that received nCPCs demonstrated improved tricuspid annular plane systolic excursion (TAPSE) and right ventricular ejection fraction (RVEF) compared to controls, and improvement was maintained to 4 weeks.

CPCs were first clinically evaluated in the phase I, randomized, open-label Cardiac Stem Cell Infusion in Patients with Ischemic Cardiomyopathy (SCIPIO, NCT00474461) trial, in which CPCs were isolated from right atrial myocardium collected from adult patients with ischemic cardiomyopathy (LVEF $\leq 40 \%$ ) during coronary artery bypass grafting (CABG). After in vitro expansion, the autologous CPCs were infused into the vessel(s) and/or graft(s) supplying previously ischemic myocardium (42). Cardiac function, as measured by LVEF, improved over the 1 -year follow-up period (increasing from $28 \%$ to $41 \%$ ), and the infarct size decreased by an average of approximately $40 \%$. These were the largest clinical improvements ever reported for cell-based therapies. Since the SCIPIO trial was not designed to demonstrate efficacy, these encouraging results warrant the pursuit of larger trials aimed at confirming the functional effects of therapeutic CPC preparations.

\section{Preclinical models of stem cell therapy}

The majority of animal studies investigating the therapeutic efficacy of stem cells do so in models that induce dysfunction of the LV, most commonly by coronary obstruction and MI. While coronary insufficiency is relatively uncommon among CHD patients, the injurious effects of myocardial ischemia and the subsequent remodeling are, nevertheless, quite useful in evaluating the effectiveness of a putative regenerative therapy. However, there are some important large animal studies of stem cell therapy that better mimic, but not completely, the RV dysfunction present in single ventricle CHD by experimentally inducing RV pressure and/or volume overload.

In one such model, RV volume overload was created in a 4-month-old sheep by placing a transannular patch from the RV outflow tract onto the main PA (26). Autologous UCB-derived mononuclear cells or control medium was then intramyocardially injected into the RV free wall. At 3 months after injection, cell-treated sheep exhibited 
significantly increased RV diastolic function (as measured by pressure-volume loops under dobutamine stress) as compared to controls. In a different ovine model, RV pressure overload was induced in 1-week-old sheep by banding of the main PA. Human cord blood stem cells or inert control medium was then injected into the RV myocardium. One month after the operation, invasive conductance catheter-based measurements were performed. Cell-treated sheep demonstrated improved RV function, as reflected by higher end-systolic elastance and preloadrecruitable stroke work, compared to controls (43).

Stem cell therapies have also been successfully studied in porcine models of pressure overload-induced $\mathrm{RV}$ dysfunction secondary to PA banding. In one such model, immunosuppressed juvenile Yorkshire swine underwent PA banding followed immediately by intramyocardial injection of 1 million human MSCs or inert control medium $(44,45)$. Four weeks following banding and injection, MSC-treated pigs exhibited less RV dilation (as measured by RV enddiastolic area and RV end-systolic area) and improved RV function (as measured by RV fractional area change and $\mathrm{RV}$ strain) compared to controls (45). A subsequent experiment evaluating the efficacy of 1 million human CPCs in the same model yielded similar attenuation of RV dilation, as well as improvement in RV function (44).

Even in the setting of favorable functional outcomes, preclinical studies are almost universally characterized by minimal retention/differentiation of exogenous stem cells $(43,44)$. These results have driven a shift in paradigm from one based on stem cell engraftment and differentiation to one that focuses on paracrine pathways. The specific pathways involved are an area of active research, but animal models of stem cell therapy have yielded important insights into some of the fundamental tissue-level mechanisms. Pigs treated with CPCs following PA banding showed less fibrosis and increased vascular density in the RV free wall compared to controls (44). A similar increase in vascular density was observed in the previously mentioned ovine models of RV dysfunction. At the molecular level, MSC treatment following PA banding was associated with less RV hypertrophy, as well as greater expression of growth differentiation factor 15 (GDF-15) and its downstream effector, SMAD 2/3. These findings provide evidence that MSCs may promote expression of GDF-15 within the myocardium, triggering a pathway which has been shown to inhibit hypertrophy (45). These findings support the notion that, while stem cells themselves do not engraft and differentiate in significant numbers, they do interact with their local cellular environment to promote favorable ventricle remodeling.

\section{Clinical trials of stem cell therapy in children}

Relative to the many completed and ongoing adult trials, some of which were discussed along with their respective stem cell types, there are a small but growing number of clinical trials of stem cell therapy for patients with CHD. The majority of these trials focus on univentricular CHD (Table 1), but they differ in the choice of stem cell type, time of administration, and method of administration. One of the first such trials was the Transcoronary Infusion of Cardiac Progenitor Cells in Patients with Single Ventricle Physiology Trial (TICAP, NCT01273857) a phase I nonrandomized, controlled trial aimed at demonstrating the safety of intracoronary delivery of CDCs in latepalliation single ventricle patients (46). In this study, patients underwent administration of autologous CDCs by intracoronary injection 4 to 5 weeks after the stage II palliative or the stage III palliative operation. Ventricular function was measured by echocardiography at 24, 30, and 36 weeks, and an additional function assessment by cardiac magnetic resonance imaging (MRI) at 36 weeks. Cardiac catheterization was also performed at 30-36 weeks. No adverse events (procedural complications, life-threatening arrhythmia, myocardial necrosis, sudden death) were reported in the CDC-treated cohort $(\mathrm{n}=7)$. At 18 months of follow-up, the CDC-treated cohort demonstrated an improvement in RVEF $(46.9 \% \pm 4.6 \%$ to $54.0 \% \pm 2.8 \%)$ and a significant reduction in tricuspid valve annulus diameter, while control patients showed negligible functional improvement (RVEF $46.7 \% \pm 4.4 \%$ to $48.7 \% \pm 6.7 \%$ ), and no change in diameter of the tricuspid valve annulus. CDCtreated patients also showed significant reductions in RV hypertrophy, as reflected by RV free wall mass, and weightindexed end-systolic and end-diastolic volumes. The somatic growth of CDC-treated patients, as reflected by z scores for height and weight, also significantly accelerated in the first 18 months of the trial, while there was no such change in the control group.

The TICAP trial was followed by the phase II randomized, controlled Cardiac Progenitor Cell Infusion to Treat Univentricular Heart Disease trial (PERSEUS, NCT01829750). Similar to TICAP, CDCs were administered by intracoronary injection following either the stage II or stage III palliative operations (47). The primary end-point was change in ventricular ejection fraction from 


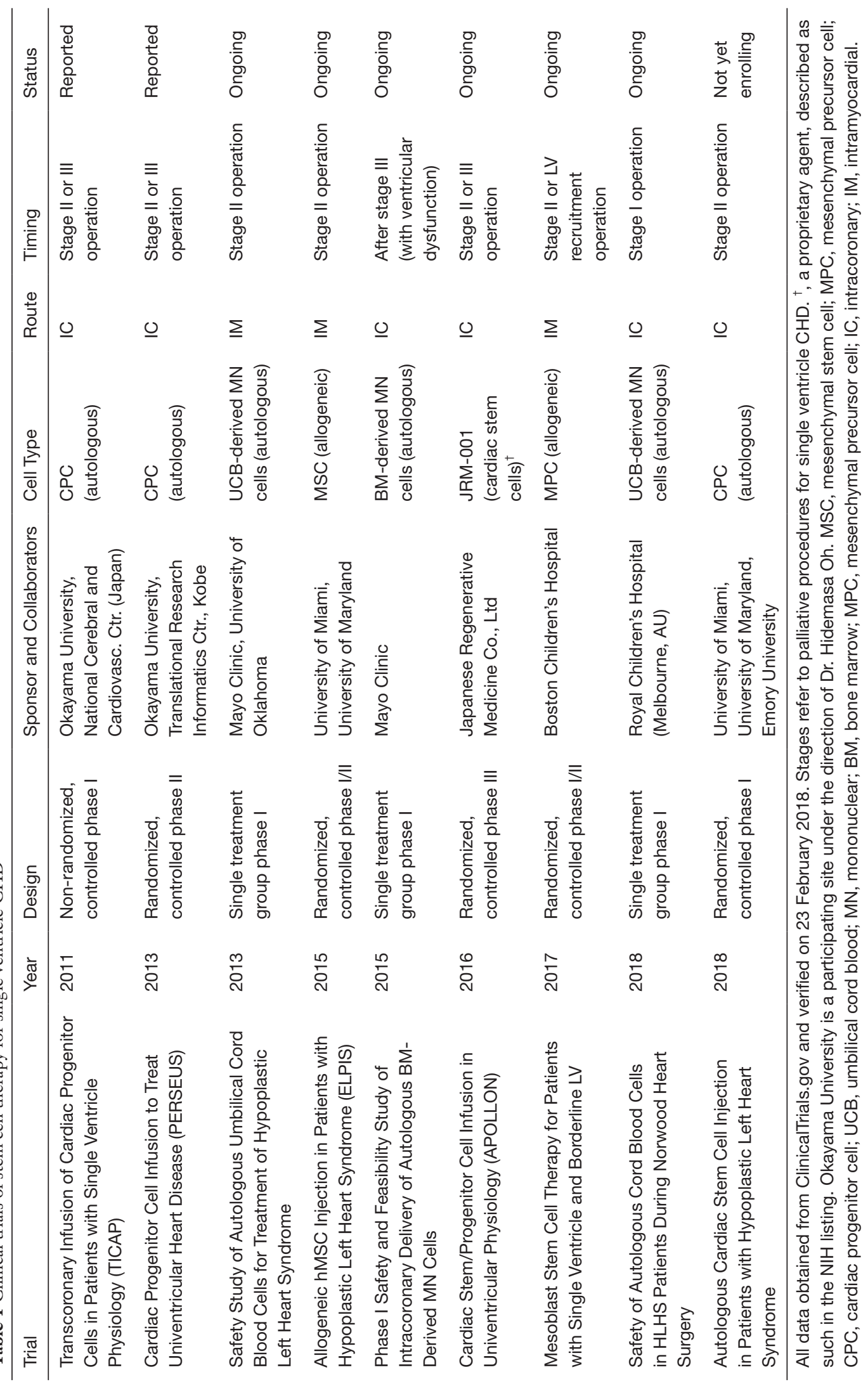




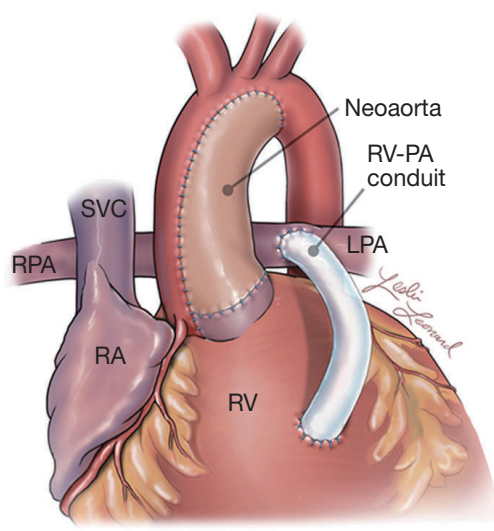

Norwood procedure

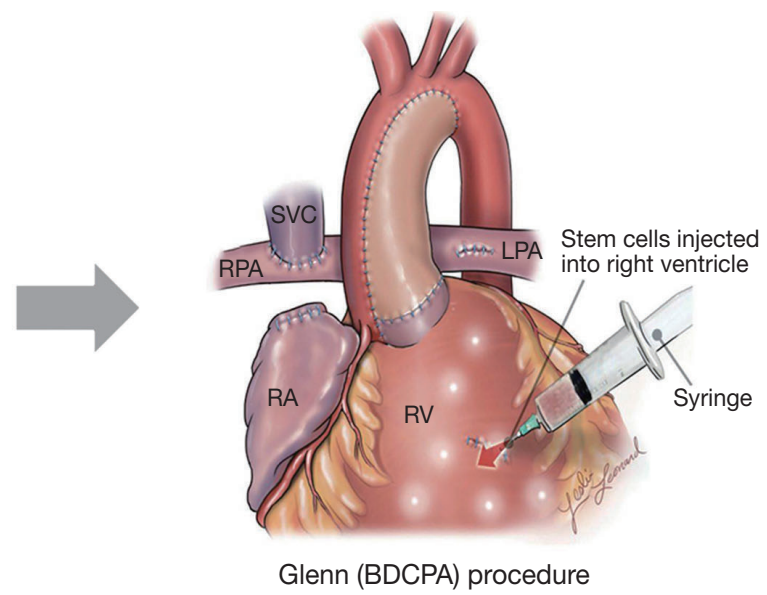

Figure 1 Cardiac anatomy following the Norwood operation (stage I) and Glenn operation (stage II) for the palliation of HLHS. With one exception, all current trials of stem cell therapy for HLHS provide cell administration at the time of or shortly after the stage II or stage III operations (Fontan, not shown). Some trials (e.g., TICAP and PERSEUS) were designed around catheter-delivered cell preparations, while others (e.g., ELPIS) utilize direct intramyocardial cell injection as shown in the Glenn illustration. Adapted with permission from Wehman B, Siddiqui OT, Mishra R, et al. Stem Cell Therapy for CHD: Towards Translation. Cardiol Young 2015;25:58-66. HLHS, hypoplastic left heart syndrome. SVC, superior vena cava; RPA, right pulmonary artery; RA, right atrium; RV, right ventricle ; LPA, left pulmonary artery ; RV$\mathrm{PA}$, right ventricle to pulmonary artery.

baseline to 3 months, and was measured by cardiac MRI, echocardiography, and ventriculography. The CDCtreated group showed greater improvement in ventricular function at 3 months than controls (6.4\% versus $1.3 \%)$, and this effect was persistent at 1 year. CDC therapy was also associated with improved quality of life at 1 year postinjection. Follow-up analysis at 36 months demonstrated sustained improvement in ventricular ejection fraction among patients having received CDCs (8.0\% vs. $2.2 \%)$ (48). Stem cell therapy was also associated with decreased serum $\mathrm{B}$-type natriuretic peptide (BNP) levels and higher age-toweight z-score.

In addition to these two seminal trials, several additional studies evaluating stem cell therapy in single ventricle patients are underway (Table 1). The phase III Cardiac Stem/Progenitor Cell Infusion in Univentricular Physiology (APOLLON, NCT02781922) trial builds on the TICAP and PERSEUS trials, further evaluating the efficacy of intracoronary delivery of autologous CDCs in single ventricle patients. Other trials are evaluating autologous UCB-derived mononuclear cells intervening at the time of stage II operation in HLHS patients, and autologous bone marrow-derived mononuclear cells after stage III operation. Allogeneic stem cell preparations are also making their way into clinical trials, such as the phase I/II Allogeneic Human MSC Injection in Patients with Hypoplastic Left Hearts
(ELPIS, NCT02398604) trial, an ongoing investigation of the safety and efficacy of intramyocardially injected allogeneic MSCs at the time of the stage II operation in HLHS patients (Figure 1) (49).

\section{Opportunities for clinical progress}

Optimization of the timing, method of delivery, and composition of stem cell-based therapeutics will be fundamental to their broad clinical implementation. Although the pediatric clinical experience with these variables remains sparse, preclinical and adult clinical trials do provide valuable, translatable insights.

The timing of stem cell administration is of tremendous importance in patients with acute myocardial insults (e.g., MI). In a rodent MI model, 2 million MSCs were delivered transepicardially, either immediately following or one week after MI. At 4 weeks follow-up, greater preservation of LVEF was found in the group that received MSCs at the time of MI induction (50). In human patients, acute expansion and activation of c-kit ${ }^{+}$CPCs following MI has been demonstrated (51). Activation and proliferation of these CPCs following injury are likely transient, and may be temporally related to the kinetics of endogenous myocardial repair pathways, including differentiation of resident cardiac stem or progenitor cells. Transplanted stem cells may be 
most effective in supporting pathways that the endogenous myocardium activates at the time of injury or stress, rather than acting as the primary stimulators of these pathways at a later time point.

In spite of the more protracted course of $\mathrm{RV}$ deterioration in patients with single ventricle CHD, timing will remain an important consideration. Progression towards right ventricular dysfunction and failure in these patients may take multiple decades, which, in conjunction with the multi-staged nature of surgical palliation for HLHS, provides many time points at which stem cellbased interventions could be considered. There is evidence, however, that stem cell-based therapies for single ventricle CHD may be most effective when applied early in life, that is, early in the progression of ventricular dysfunction and during the period of most active regeneration. Although the human myocardium is often characterized as a postmitotic organ, recent evidence suggests that low levels of cardiomyocyte proliferation exist in the young myocardium, which declines with advancing age $(52,53)$. Measurement of carbon-14 incorporation reveals renewal of cardiomyocytes at a rate of $1 \%$ annually at approximately age 20 , and $0.3 \%$ at 75 (53). In addition, levels of endogenous c-kit ${ }^{+} \mathrm{CPCs}$ are highest in neonates and rapidly decline with age (38), and, as previously mentioned, neonatal derived CPCs have a significantly higher in vitro proliferative capacity, cloning efficiency, and resistance to telomere attrition compared to adult derived CPCs (40). Both stimulation of endogenous cardiomyocyte proliferation and activation of resident cardiac stem and progenitor cells have been implicated in stem cell mediated recovery of the injured myocardium. If stem cell based therapies are most effective when they further stimulate endogenously active pathways, the progressive decline of cardiomyocyte proliferation and resident CPC abundance and potency with advancing age would suggest that earlier stem cell based interventions may be more successful in attenuating dysfunction and failure of the single RV. Indeed, follow-up analysis of the TICAP trial demonstrated a strong association between younger age at the time of administration and greater improvement in RVEF, providing further support for the critical window within the early lifetime for HLHS patients (48). Although the Norwood is the first of three staged palliative procedures, administration of stem cells during this operation or prior to the stage II procedure may be difficult because of the morbidities that often arise during this challenging period. Therefore, while cell therapy may eventually be shown to be most effective early in life, the stage II or III palliative operations may be an ideal time point to evaluate stem cell safety in HLHS patients, as they tend to be performed in a time of relative clinical stability.

The method of stem cell administration is another important factor to consider, both in terms of procedural safety and functional efficacy. The TICAP trial demonstrated safety of intracoronary delivery of CDCs using a stop-flow method (48). However, the physical size and necessary dosage of other stem cell types should be considered in light of possible occlusion of the microvasculature following intracoronary injection. Intravascular delivery of MSCs (particularly large stem cells) has been shown to cause detectable microvascular obstruction in both murine and porcine models of MI with reperfusion $(54,55)$. The route of administration may also affect the efficacy of stem cell treatments by influencing the primary pathways involved in mediating recovery. Direct comparisons of stem cell administration methods have not been conducted in clinical trials for CHD patients. However, in adult patients with dilated cardiomyopathy, retention of $\mathrm{CD} 34^{+}$cells and functional recovery was found to be superior with transendocardial delivery compared to intracoronary administration $(56,57)$. On the other hand, in a porcine model of MI with reperfusion, intracoronary delivery of adipose tissue-derived stem cells (ADSCs) mediated greater neovascularization than transendocardial delivery, despite similar levels of engraftment and differentiation in both groups $(57,58)$. A comparison of administration routes in animal models of induced $\mathrm{RV}$ dysfunction would be valuable in providing insights for optimal delivery of stem cells for CHD patients.

Finally, as our understanding of the cellular mechanisms underlying stem cell-based therapy advances, we will continue to refine the composition of our stem cellbased therapeutics. Since the current paradigm for stem cell therapy emphasizes the paracrine mechanisms by which exogenous stem cells triggers regenerative and cardioprotective pathways within the recipient myocardium, much of the investigative focus has shifted away from the cells themselves and into the changes induced in the myocardium (e.g., angiogenesis, cell survival, attenuation of hypertrophy, differentiation of endogenous stem cells) $(45,59)$. By working backwards from these components of favorable remodeling, the stem cell secretome can be assayed for candidate substances that are expected to exert these effects. Such an analysis can be effectively performed in silico (using in vitro or in vivo outcome data as inputs), and has identified a number of molecules that are critical 
Table 2 Proteins identified in the secretome of neonatal cardiac progenitor cells and the respective pathways involved

\begin{tabular}{ll}
\hline Canonical pathways & Proteins \\
\hline Protein Ubiquitination & HSPD1, PSMA3, HSPE1, UBE2D2, PSMD4, PSMA7, PSMD9, HSPA8, PSMB7, SKP1, TCEB1, PSMB3, \\
Pathway & PSMA1, HSP90AA1, PSMA5, USP14, PSMC3, UBE2N, PSME1, USO1, HSPA, HLA-A, PSMB4, UBE2I, \\
mTOR Signaling & EIF3G, RPS10, EIF4A2, EIF3B, RPS5, RPS20, PPP2R1A, EIF4A1, EIF4EBP1, VEGFC, EIF3J, EIF4G1, \\
& PPP2CA, EIF3A, RPS7, RPS2, PDGFC, RPS3, RPS12, RPSA, RPS16, MAPK1, EIF3K, RPS4X, EIF3C, EIF3F \\
ERK/MAPK Signaling & TLN1, CRKL, PPP1R12A, ITGA2, PPP1R7, PPP1CA, PXN, YWHAH, MAPK1, PPP2R1A, CRK, EIF4EBP1, \\
& PPP2CA \\
HIF-1 $\alpha$ Signaling & MMP3, MMP9, TCEB1, MAPK1, MMP10, HSP90AA1, APEX1, VEGFC, PDGFC \\
PI3K/AKT Signaling & GDF15, ITGA2, PPP2R1A, MAPK1, HSP90AB1, HSP90AA1, YWHAH, EIF4EBP1, PPP2CA \\
HIPPO Signaling & PPP1R12A, PPP1R7, PPP1CA, SKP1, PPP2R1A, YAP1, YWHAH, PPP2CA \\
VEGF Signaling & EIF2S1, EIF2S2, ACTG1, PXN, MAPK1, EIF1, VEGFC, PDGFC \\
Neuregulin Signaling & CRKL, ITGA2, MAPK1, CRK, HSP90AB1, HSP90AA1 \\
Telomerase Signaling & PTGES3, PPP2R1A, MAPK1, HSP90AB1, HSP90AA1, PPP2CA \\
Antiproliferative Role of & TGFB1, SKP1, PABPC1, MAPK1 \\
\hline TOB in T Cell Signaling & \\
\hline
\end{tabular}

to stem cell efficacy (40). As myocardial regeneration and recovery is a multifaceted process, however, it is possible that failing single ventricle patients would benefit from the administration of multiple cell types at different time points, or perhaps even coadministration of multiple cells at a single time point. In a rat MI model, coinjection of MSCs and c-kit ${ }^{+}$CPCs was found to be more effective in mediating recovery when compared to administration of either cell type alone (60). The authors attributed this to a synergistic effect which improves retention of transplanted cells, and a therefore greater paracrine secretion of proangiogenic factors. In addition to the stem cell type and method of isolation, donor characteristics may influence the therapeutic efficacy of derived cells. This has been conclusively demonstrated with regard to donor age, as described in the Preclinical Models of Stem Cell Therapy section with regard to neonatal and adult CPCs. This same study found that neonatal CPC-derived total conditioned medium (nTCM) outperformed adult CPC-derived total conditioned media (aTCM) in mediating post MI recovery in rats. It may be valuable to explore the age dependence of other cell types that may be used in clinical applications in CHD patients, particularly when allogeneic preparations are considered $(38,40)$. Consistent with the paracrine model of myocardial recovery, we have reported that injecting a single dose of the total conditioned media from neonatal CPCs into a rodent $\mathrm{MI}$ model outperformed the transplantation of live neonatal CPCs with respect to functional recovery (40). Proteins found in the secretome of neonatal CPCs affect a variety of pathways that may play important roles in cardiac regeneration, as identified by Ingenuity Pathway Analysis (IPA, Qiagen, Hilden, Germany) (Table 2). Injection of the purified exosomal component of the TCM into the same model yielded functional recovery similar to that achieved using whole cells. The therapeutic efficacy of stem cell derived exosomes, which are secreted nanovesicles that contain microRNAs and proteins, has previously been demonstrated (61). It may therefore be possible to design stem cell-based therapies around these secreted products rather than live cells.

\section{Conclusions}

There is growing evidence supporting the therapeutic potential of stem cell administration in children with CHD, especially those with single ventricle physiology. Preclinical studies have verified the benefits of various stem cell types in animal models relevant to CHD, and have identified the secretome as the functional unit of various stem cell types. While a small but growing number of clinical trials in CHD patients have shown stem cell-dependent improvements in ventricular function and quality of life, further investigation will define the ideal timing, dosing, and stem cell type or types for the treatment of CHD patients. Given the 
inherent vitality of the young myocardium and their early stage in the development of heart failure, children may indeed be the best responders to cell therapy. As the field continues to evolve, the application of stem cell-based therapeutics for myocardial protection and regeneration in CHD patients will continue to be refined, marching ever closer to a powerful treatment for these challenging conditions.

\section{Acknowledgements}

Funding: This work was supported by the National Institutes of Health grant numbers (2T32AR007592-21 to GJ Bittle, 1R01HL118491 and 5R01HL139060-02 to S Kaushal); MedImmune; and the Maryland Stem Cell Research Fund.

\section{Footnote}

Conflicts of Interest: The authors have no conflicts of interest to declare.

\section{References}

1. Fixler DE, Nembhard WN, Salemi JL, et al. Mortality in first 5 years in infants with functional single ventricle born in Texas, 1996 to 2003. Circulation 2010;121:644-50.

2. Gordon BM, Rodriguez S, Lee M, et al. Decreasing number of deaths of infants with hypoplastic left heart syndrome. J Pediatr 2008;153:354-8.

3. Reller MD, Strickland MJ, Riehle-Colarusso T, et al. Prevalence of congenital heart defects in metropolitan Atlanta, 1998-2005. J Pediatr 2008;153:807-13.

4. Algra SO, Breur JM, Evens FC, et al. Improving surgical outcome following the Norwood procedure. Neth Heart J 2011;19:369-72.

5. Si MS, Pearson GD, Ohye RG. Shunt choice in single right ventricle patients: an update. Expert Rev Cardiovasc Ther 2013;11:1691-700.

6. Ohye RG, Schranz D, D'Udekem Y. Current Therapy for Hypoplastic Left Heart Syndrome and Related Single Ventricle Lesions. Circulation 2016;134:1265-79.

7. Friedberg MK, Redington AN. Right versus left ventricular failure: differences, similarities, and interactions. Circulation 2014;129:1033-44.

8. Reddy S, Bernstein D. Molecular Mechanisms of Right Ventricular Failure. Circulation 2015;132:1734-42.

9. Wilder TJ, McCrindle BW, Phillips AB, et al. Survival and right ventricular performance for matched children after stage-1 Norwood: Modified Blalock-Taussig shunt versus right-ventricle-to-pulmonary-artery conduit. J Thorac Cardiovasc Surg 2015;150:1440-50, 1452.e1-8; discussion 1450-2.

10. Yabrodi M, Mastropietro CW. Hypoplastic left heart syndrome: from comfort care to long-term survival. Pediatr Res 2017;81:142-9.

11. Winter MM, Bouma BJ, Groenink M, et al. Latest insights in therapeutic options for systemic right ventricular failure: a comparison with left ventricular failure. Heart 2009;95:960-3.

12. Bernstein D, Naftel D, Chin C, et al. Outcome of listing for cardiac transplantation for failed Fontan: a multiinstitutional study. Circulation 2006;114:273-80.

13. Deal BJ, Jacobs ML. Management of the failing Fontan circulation. Heart 2012;98:1098-104.

14. Pittenger MF, Mackay AM, Beck SC, et al. Multilineage potential of adult human mesenchymal stem cells. Science 1999;284:143-7.

15. Gnecchi M, He H, Noiseux N, et al. Evidence supporting paracrine hypothesis for Akt-modified mesenchymal stem cell-mediated cardiac protection and functional improvement. FASEB J 2006;20:661-9.

16. Du YY, Zhou SH, Zhou T, et al. Immuno-inflammatory regulation effect of mesenchymal stem cell transplantation in a rat model of myocardial infarction. Cytotherapy 2008;10:469-78.

17. Aggarwal S, Pittenger MF. Human mesenchymal stem cells modulate allogeneic immune cell responses. Blood 2005; 105:1815-22.

18. Hare JM, Traverse JH, Henry TD, et al. A randomized, double-blind, placebo-controlled, dose-escalation study of intravenous adult human mesenchymal stem cells (prochymal) after acute myocardial infarction. J Am Coll Cardiol 2009;54:2277-86.

19. Hare JM, Fishman JE, Gerstenblith G, et al. Comparison of allogeneic vs autologous bone marrow-derived mesenchymal stem cells delivered by transendocardial injection in patients with ischemic cardiomyopathy: the POSEIDON randomized trial. JAMA 2012;308:2369-79. Erratum in: JAMA 2013;310:750.

20. Hare JM, DiFede DL, Rieger AC, et al. Randomized Comparison of Allogeneic Versus Autologous Mesenchymal Stem Cells for Nonischemic Dilated Cardiomyopathy: POSEIDON-DCM Trial. J Am Coll Cardiol 2017;69:526-37.

21. Gluckman E. Current status of umbilical cord blood 
hematopoietic stem cell transplantation. Exp Hematol 2000;28:1197-205.

22. Lee OK, Kuo TK, Chen WM, et al. Isolation of multipotent mesenchymal stem cells from umbilical cord blood. Blood 2004;103:1669-75.

23. Berger MJ, Adams SD, Tigges BM, et al. Differentiation of umbilical cord blood-derived multilineage progenitor cells into respiratory epithelial cells. Cytotherapy 2006;8:480-7.

24. Nishiyama N, Miyoshi S, Hida N, et al. The significant cardiomyogenic potential of human umbilical cord bloodderived mesenchymal stem cells in vitro. Stem Cells 2007;25:2017-24.

25. Leor J, Guetta E, Feinberg MS, et al. Human umbilical cord blood-derived CD133+ cells enhance function and repair of the infarcted myocardium. Stem Cells 2006;24:772-80. Erratum in: Stem Cells 2006;24:1627.

26. Yerebakan C, Sandica E, Prietz S, et al. Autologous umbilical cord blood mononuclear cell transplantation preserves right ventricular function in a novel model of chronic right ventricular volume overload. Cell Transplant 2009;18:855-68.

27. Ma N, Stamm C, Kaminski A, et al. Human cord blood cells induce angiogenesis following myocardial infarction in NOD/scid-mice. Cardiovasc Res 2005;66:45-54.

28. Bartolucci J, Verdugo FJ, González PL, et al. Safety and Efficacy of the Intravenous Infusion of Umbilical Cord Mesenchymal Stem Cells in Patients With Heart Failure: A Phase 1/2 Randomized Controlled Trial (RIMECARD Trial [Randomized Clinical Trial of Intravenous Infusion Umbilical Cord Mesenchymal Stem Cells on Cardiopathy]). Circ Res 2017;121:1192-204.

29. Barile L, Gherghiceanu M, Popescu LM, et al. Human cardiospheres as a source of multipotent stem and progenitor cells. Stem Cells Int 2013;2013:916837.

30. Chimenti I, Smith RR, Li TS, et al. Relative roles of direct regeneration versus paracrine effects of human cardiosphere-derived cells transplanted into infarcted mice. Circ Res 2010;106:971-80.

31. Malliaras K, Li TS, Luthringer D, et al. Safety and efficacy of allogeneic cell therapy in infarcted rats transplanted with mismatched cardiosphere-derived cells. Circulation 2012;125:100-12.

32. Kanazawa H, Tseliou E, Malliaras K, et al. Cellular postconditioning: allogeneic cardiosphere-derived cells reduce infarct size and attenuate microvascular obstruction when administered after reperfusion in pigs with acute myocardial infarction. Circ Heart Fail 2015;8:322-32.

33. Makkar RR, Smith RR, Cheng K, et al. Intracoronary cardiosphere-derived cells for heart regeneration after myocardial infarction (CADUCEUS): a prospective, randomised phase 1 trial. Lancet 2012;379:895-904.

34. Chakravarty T, Makkar RR, Ascheim DD, et al. ALLogeneic Heart STem Cells to Achieve Myocardial Regeneration (ALLSTAR) Trial: Rationale and Design. Cell Transplant 2017;26:205-14.

35. Tallini YN, Greene KS, Craven M, et al. c-kit expression identifies cardiovascular precursors in the neonatal heart. Proc Natl Acad Sci U S A 2009;106:1808-13.

36. Tang XL, Rokosh DG, Guo Y, et al. Cardiac progenitor cells and bone marrow-derived very small embryonic-like stem cells for cardiac repair after myocardial infarction. Circ J 2010;74:390-404.

37. Wehman B, Sharma S, Mishra R, et al. Pediatric EndStage Failing Hearts Demonstrate Increased Cardiac Stem Cells. Ann Thorac Surg 2015;100:615-22.

38. Mishra R, Vijayan K, Colletti EJ, et al. Characterization and functionality of cardiac progenitor cells in congenital heart patients. Circulation 2011;123:364-73. Erratum in: Circulation 2012;125:e958.

39. He JQ, Vu DM, Hunt G, et al. Human cardiac stem cells isolated from atrial appendages stably express c-kit. PLoS One 2011;6:e27719.

40. Sharma S, Mishra R, Bigham GE, et al. A Deep Proteome Analysis Identifies the Complete Secretome as the Functional Unit of Human Cardiac Progenitor Cells. Circ Res 2017;120:816-34.

41. Agarwal U, Smith AW, French KM, et al. Age-Dependent Effect of Pediatric Cardiac Progenitor Cells After Juvenile Heart Failure. Stem Cells Transl Med 2016;5:883-92.

42. Bolli R, Chugh AR, D'Amario D, et al. Cardiac stem cells in patients with ischaemic cardiomyopathy (SCIPIO): initial results of a randomised phase 1 trial. Lancet 2011;378:1847-57.

43. Davies B, Elwood NJ, Li S, et al. Human cord blood stem cells enhance neonatal right ventricular function in an ovine model of right ventricular training. Ann Thorac Surg 2010;89:585-93, 593.e1-4.

44. Wehman B, Pietris N, Bigham G, et al. Cardiac Progenitor Cells Enhance Neonatal Right Ventricular Function After Pulmonary Artery Banding. Ann Thorac Surg 2017;104:2045-53.

45. Wehman B, Sharma S, Pietris N, et al. Mesenchymal stem cells preserve neonatal right ventricular function in a porcine model of pressure overload. Am J Physiol Heart Circ Physiol 2016;310:H1816-26.

46. Ishigami S, Ohtsuki S, Tarui S, et al. Intracoronary 
autologous cardiac progenitor cell transfer in patients with hypoplastic left heart syndrome: the TICAP prospective phase 1 controlled trial. Circ Res 2015;116:653-64.

47. Ishigami S, Ohtsuki S, Eitoku T, et al. Intracoronary Cardiac Progenitor Cells in Single Ventricle Physiology: The PERSEUS (Cardiac Progenitor Cell Infusion to Treat Univentricular Heart Disease) Randomized Phase 2 Trial. Circ Res 2017;120:1162-73.

48. Tarui S, Ishigami S, Ousaka D, et al. Transcoronary infusion of cardiac progenitor cells in hypoplastic left heart syndrome: Three-year follow-up of the Transcoronary Infusion of Cardiac Progenitor Cells in Patients With Single-Ventricle Physiology (TICAP) trial. J Thorac Cardiovasc Surg 2015;150:1198-207, 1208.e1-2.

49. Kaushal S, Wehman B, Pietris N, et al. Study design and rationale for ELPIS: A phase I/IIb randomized pilot study of allogeneic human mesenchymal stem cell injection in patients with hypoplastic left heart syndrome. Am Heart J 2017;192:48-56.

50. Richardson JD, Bertaso AG, Psaltis PJ, et al. Impact of timing and dose of mesenchymal stromal cell therapy in a preclinical model of acute myocardial infarction. J Card Fail 2013;19:342-53.

51. Urbanek K, Torella D, Sheikh F, et al. Myocardial regeneration by activation of multipotent cardiac stem cells in ischemic heart failure. Proc Natl Acad Sci U S A 2005;102:8692-7.

52. Parmacek MS, Epstein JA. Cardiomyocyte renewal. N Engl J Med 2009;361:86-8.

53. Bergmann O, Bhardwaj RD, Bernard S, et al. Evidence for cardiomyocyte renewal in humans. Science 2009;324:98-102.

54. Freyman T, Polin G, Osman H, et al. A quantitative,

Cite this article as: Ambastha C, Bittle GJ, Morales D, Parchment N, Saha P, Mishra R, Sharma S, Vasilenko A, Gunasekaran M, Al-Suqi MT, Li D, Yang P, Kaushal S. Regenerative medicine therapy for single ventricle congenital heart disease. Transl Pediatr 2018;7(2):176-187. doi: 10.21037/ tp.2018.04.01 randomized study evaluating three methods of mesenchymal stem cell delivery following myocardial infarction. Eur Heart J 2006;27:1114-22.

55. Furlani D, Ugurlucan M, Ong L, et al. Is the intravascular administration of mesenchymal stem cells safe? Mesenchymal stem cells and intravital microscopy. Microvasc Res 2009;77:370-6.

56. Vrtovec B, Poglajen G, Lezaic L, et al. Comparison of transendocardial and intracoronary CD34+ cell transplantation in patients with nonischemic dilated cardiomyopathy. Circulation 2013;128:S42-9.

57. Golpanian S, Schulman IH, Ebert RF, et al. Concise Review: Review and Perspective of Cell Dosage and Routes of Administration From Preclinical and Clinical Studies of Stem Cell Therapy for Heart Disease. Stem Cells Transl Med 2016;5:186-91.

58. Rigol M, Solanes N, Farré J, et al. Effects of adipose tissue-derived stem cell therapy after myocardial infarction: impact of the route of administration. J Card Fail 2010;16:357-66.

59. Mirotsou M, Jayawardena TM, Schmeckpeper J, et al. Paracrine mechanisms of stem cell reparative and regenerative actions in the heart. J Mol Cell Cardiol 2011;50:280-9.

60. Bao L, Meng Q, Li Y, et al. C-Kit Positive Cardiac Stem Cells and Bone Marrow-Derived Mesenchymal Stem Cells Synergistically Enhance Angiogenesis and Improve Cardiac Function After Myocardial Infarction in a Paracrine Manner. J Card Fail 2017;23:403-15.

61. Mentkowski KI, Snitzer JD, Rusnak S, et al. Therapeutic Potential of Engineered Extracellular Vesicles. AAPS J 2018;20:50. 